\title{
Dynamics of the Electric Field in a GaAs/AlGaAs Superlattice after Femtosecond Optical Excitation: Application of Time-Resolved Spectroscopic Techniques
}

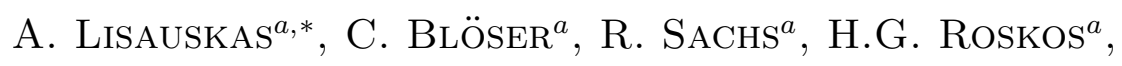
A. JuOzapavičiuss ${ }^{b}$, G. VAlušis ${ }^{a, b}$ And K. KÖHLER $^{c}$

${ }^{a}$ Physikalisches Institut, J.W. Goethe-Universität, Frankfurt/Main 60054, Germany

${ }^{b}$ Semiconductor Physics Institute, Vilnius 01108, Lithuania

${ }^{c}$ Fraunhofer-Institut für Angewandte Festkörperphysik, Freiburg 79108, Germany

We apply time-resolved photocurrent and differential electroreflectance spectroscopy to study the evolution of the internal field in a GaAs/AlGaAs superlattice after pulsed optical excitation at low temperature. The electric field dynamics is investigated by tracing the spectral position of the Wannier-Stark transitions as a function of delay time. We determine the electron sweep-out time, extract detailed information about the picosecond-time-scale drift of the charge carriers by comparing the measured data with the results of semi-classical self-consistent model calculations, and evaluate the two experimental techniques with respect to their ability to provide information about the carrier and field dynamics.

PACS numbers: 03.65.Sq, 78.47.+p

\section{Introduction}

Semiconductor superlattices placed in an electric field make an excellent environment to observe a large variety of phenomena related to charge carriers in periodic potentials including Bloch oscillations [1, 2], miniband collapse [3], Zener tunneling [4], etc. By varying the applied electric field, superlattices can be tuned

*corresponding author; e-mail: lisauskas@physik.uni-frankfurt.de 
to a negative-differential-velocity regime and can thus be used as active components in high-frequency oscillators [5]. As a rule, in order to realize an electrically driven device, one needs a certain degree of doping. Introduction of charge carriers by doping is very convenient technologically and well-suited for circuit designers. However, doped superlattices exhibit the formation of electric-field domains under external fields [6]. Therefore, an understanding of the evolution of the electric field is an important issue with a considerable practical interest. The field dynamics can be traced by electrical means, for instance, by time-resolved current measurements [6]. An alternative option to investigate the domain formation is to use optical techniques. In the latter case, physical effects can be probed on much shorter time scales, and one can investigate both electrically doped superlattices and intrinsic superlattices with optical injection of mobile electrons and holes.

In this report, we specifically concentrate ourselves on the field evolution in an optically excited intrinsic GaAs/AlGaAs superlattice. The work, apart from a practical interest, has a strong motivation coming from basic solid-state physics: we search for appropriate experimental conditions to observe the so-called Bloch gain - the phonon-assisted inversion-less amplification of terahertz radiation by optical transitions between the Wannier-Stark states in superlattices [7-10]. Since one of the necessary conditions for a successful Bloch-gain experiment is to ensure a sufficiently homogeneous electric field across the structure, we seek to estimate the time scale within which the electric-field domains begin to predominate [11].

\section{Samples and experimental techniques}

We explore an intrinsic GaAs $/ \mathrm{Al}_{0.3} \mathrm{Ga}_{0.7}$ As superlattice (SL) grown on semiinsulating GaAs substrate by molecular beam epitaxy. The SL consists of 50 periods of $6.7 \mathrm{~nm}$ wide wells and $1.7 \mathrm{~nm}$ wide barriers, and is designed to have a width $\Delta_{\mathrm{e}}$ of the first electron miniband of $33 \mathrm{meV}$. In order to apply an electric bias field, an $n$-doped buried contact layer (600 nm thick) is inserted between SL and substrate. A top contact is realized via a $3 \mathrm{~nm}$ thick semi-transparent $\mathrm{Cr} / \mathrm{Au}$ layer. For the measurements, we prepared a $2 \mathrm{~mm}$ long and $500 \mu \mathrm{m}$ wide SL waveguide structure by etching away most of the wafer from the top side down to the buried contact layer. On this layer, we then deposited an annealed $\mathrm{Au} / \mathrm{Ge} / \mathrm{Ni}$ alloy ohmic contact.

The superlattice, placed into a closed-cycle helium cryostat at $10 \mathrm{~K}$ temperature, was excited by 70 fs long pump pulses delivered by a $83 \mathrm{MHz}$ Ti:sapphire laser. Two time-resolved optical techniques were used to trace the evolution of the internal electric field: differential electroreflectance spectroscopy and photocurrent spectroscopy. In both cases, care was taken to ensure that the size of the probe spot was equal to or smaller than the size of the pumped spot. The field in the structure was determined by measuring the spectral shift of Wannier-Stark-ladder transitions. 


\section{Experimental results and discussion}

Figure 1 shows electroreflectance (Fig. 1a) and photocurrent (Fig. 1b) spectra taken without pump excitation. The spectra illustrate that both techniques allow
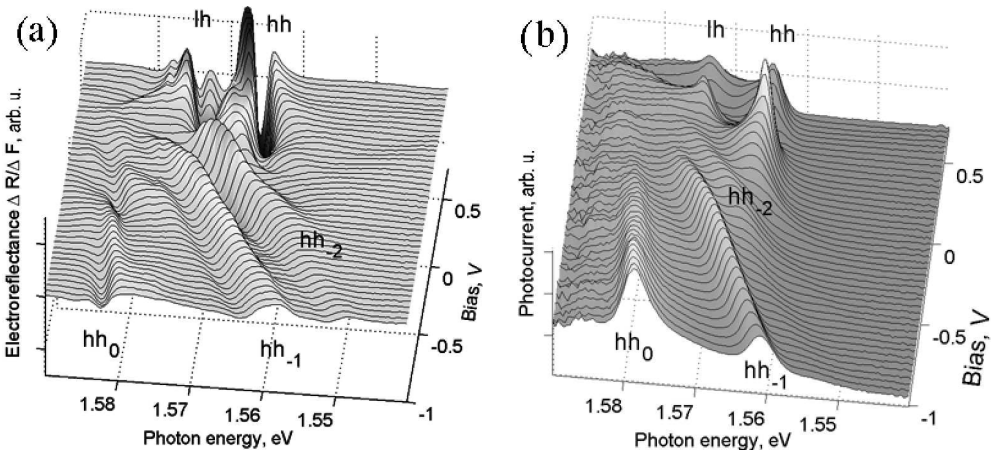

Fig. 1. Excitonic spectra measured for a GaAs/AlGaAs superlattice taken by (a) differential electroreflectance and (b) photocurrent spectroscopy.

us to identify the relevant excitonic transitions. The sensitivity to their details is different because electroreflectance - in contrast to photocurrent spectroscopy reproduces absorption lines as a second-order derivative. In the flat-band regime (applied voltage of about $0.5 \mathrm{~V}$ ), two transitions, heavy-hole $(h h)$ and light-hole $(l h)$, are clearly resolved around $1.564 \mathrm{eV}$ in both spectra. With the increase of the electric field (reducing the bias voltage), the spectra evolve into the Wannier-Stark ladder. In the figure, we mark only the main lines: $h h_{0}$ is associated with spatially direct, while $h h_{-1}, h h_{-2}$ label spatially indirect transitions. Well-resolved spectral peaks in both spectra indicate the high quality of the SL.

In the time-resolved measurements, we focused our attention on one of the most pronounced excitonic lines, $h h_{-1}$, and used it as a sensitive electric-field detector.

The experimental data are displayed in Fig. 2. Both the electroreflectance (Fig. 2a) and the photocurrent (Fig. 2b) time-resolved spectra were recorded at the same experimental conditions - at an applied voltage of $-0.85 \mathrm{~V}$ (corresponding to an internal bias field of $30 \mathrm{kV} / \mathrm{cm}$ ) and an excitation density of $4 \times 10^{15} \mathrm{~cm}^{-3}$. In both spectra, one can see a clear continuous spectral shift of the $h h_{-1}$ peak by as much as $5 \mathrm{meV}$ during a time scale of $25 \mathrm{ps}$ after excitation, later followed by a slow recovery. The shift is attributed to field screening. There is a remarkable difference, however, with respect to the direction of the observed shift. In the electroreflectance spectrum, the $h h_{-1}$ peak undergoes a red-shift, i.e., it shifts to lower photon energies. In contrast, in the photocurrent spectrum, $h h_{-1}$ moves to the blue side by $5 \mathrm{meV}$.

In order to clarify such cardinally different experimental results we have solved numerically a self-consistent set of drift-diffusion and Poisson's equations 
(a)

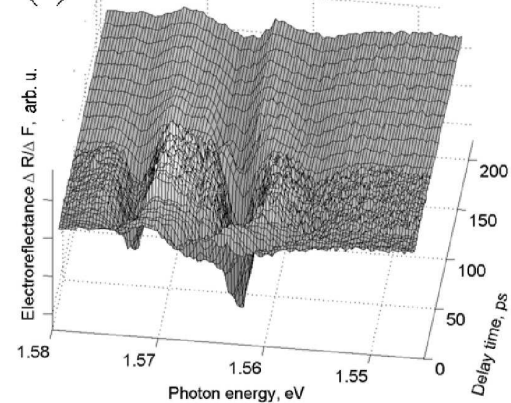

(b)

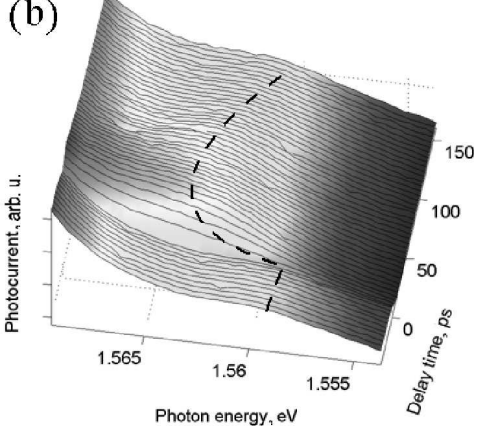

Fig. 2. Time-resolved spectra in the vicinity of the $h h_{-1}$ excitonic transition taken at an internal electric field of $30 \mathrm{kV} / \mathrm{cm}$ and a carrier density of $4 \times 10^{15} \mathrm{~cm}^{-3}$ measured using (a) differential electroreflectance and (b) photocurrent spectroscopy (the data of Fig. $2 \mathrm{~b}$ had to be taken with a different sample than those of Figs. 1a, b and 2a. The $h h_{-1}$ line was less pronounced in the second sample). The dotted line indicates the peak position of $h h_{-1}$ excitonic transition.

along the superlattice growth coordinate $z$ :

$$
\left\{\begin{array}{l}
\frac{\partial n(z, t)}{\partial t}=-\frac{\partial}{\partial z} n(z, t) \mu_{\mathrm{e}}(z, t) F(z)-\frac{k T}{q} \frac{\partial}{\partial z} \mu_{\mathrm{e}}(z, t) \frac{\partial}{\partial z} n(z, t) \\
\frac{\partial p(z, t)}{\partial t}=-\frac{\partial}{\partial z} p(z, t) \mu_{\mathrm{h}} F(z)-\frac{k T}{q} \mu_{\mathrm{h}} \frac{\partial^{2}}{\partial z} p(z, t) \\
\frac{\partial F(z, t)}{\partial z}=\frac{q}{\varepsilon \varepsilon_{0}}[p(z, t)-n(z, t)] \\
\mu_{\mathrm{e}}(z)=q \Delta_{\mathrm{e}} \tau_{\mathrm{e}} d^{2} /\left\{2+2\left[q \tau_{\mathrm{e}} d F(z)\right]^{2}\right\} .
\end{array}\right.
$$

Here, $\mu_{\mathrm{e}}$ and $\mu_{\mathrm{h}}$ are the electron and hole mobilities, $n$ and $p$ are the local electron and hole densities at the moment $t, q$ is the charge, $\tau_{\mathrm{e}}$ the momentum scattering time for electrons, and $d$ the period of SL. $k$ denotes the Boltzmann constant, $\varepsilon$ and $\varepsilon_{0}$ are the dielectric permittivities of the SL and vacuum, and $T$ is the temperature. The system was solved only for spatial points which correspond to the middle of each well. We apply the semiclassical approximation for $\mu_{\mathrm{e}}$, which is derived assuming nearest-neighbor coupling of the quantum wells of the SL [12]. For a description of the dynamics of the holes, one cannot work within a one-band model because of the strong scattering between heavy- and light-hole bands. Leaving a more complex treatment to future work, we limit ourselves here to the zero-order approximation and assume a field-independent effective mobility for the holes.

The model calculations describe the spatio-temporal behavior of the charge carriers and the electric field. In particular, at the excitation densities of our experiments, the model predicts the formation of two regions: (i) an electron-rich region with partially screened and rather homogeneous field, and (ii) an electron-depleted region with high gradient of the field. The maximal electric field inhomogeneity is reached just when the last electrons leave the SL 25 ps after photoexcitation. 
Later, with the sweep-out of the holes, the electric field restores to the initial homogeneous conditions.

Bearing this in mind, we return to the question of the direction of the line shift in our measurements. In the case of the photocurrent spectra shown in Fig. $2 \mathrm{~b}$, the blue-shift of the peak of the $h h_{-1}$ excitonic transition can be well explained as to be mainly determined by the screened region. With the field being rather constant there, the quantum wells in this region contribute oscillator strength to the absorption line at the same photon energy. The blue-shift then represents the reduction of the field compared to the initial (bias) field. In the region with high field gradient, in contrast, the photon energy of the $h h_{-1}$ transition changes from quantum well to quantum well with the local field, which only results in a broad spectral feature with low peak amplitude.

In the case of electroreflectance spectroscopy (Fig. 2a), our model calculations indicate that the differential signal is dominated by contributions from regions in the SL with strong field gradient. As these are also regions with an increased field magnitude, the electroreflectance spectra display a red-shift of the peak of $h h_{-1}$ transition.

Although both spectroscopic techniques show the build-up of dynamical field inhomogeneities, photocurrent spectroscopy appears to be more tractable due to its direct, non-differential character. A quantitative comparison of photocurrent data and model calculations for a similar superlattice as that investigated here can be found elsewhere [11]. Our key result is that the observed shift of the $h h_{-1}$ transition gives a measure for the sweep-out time of electrons and holes. The big difference of the effective masses of these species allows treating the rapid initial shift and following much slower recovery as independent from each other. We find that electrons are swept out of the superlattice within 10-30 ps, which sets a reasonable time window for future Bloch-gain measurements.

\section{Acknowledgments}

The work was supported by DFG (contract numbers RO 770/16-2, RO 770/23-1 and LI 1280/1-1) and the NATO Science for Peace Programme (SfP 978030). G. V. is grateful to the Alexander von Humboldt Foundation for the support of his research stay at Johann-Wolfgang-Goethe University.

\section{References}

[1] J. Feldmann, K. Leo, J. Shah, D.A.B. Miller, J.E. Cunnigham, S. Schmitt-Rink, T. Meier, G. von Plessen, A. Schulze, P. Thomas, Phys. Rev. B 46, 7252 (1992).

[2] C. Waschke, H.G. Roskos, R. Schwendler, K. Leo, H. Kurz, K. Köhler, Phys. Rev. Lett. 70, 3319 (1993).

[3] M. Holthaus, Phys. Rev. Lett. 69, 351 (1992). 
[4] B. Rosam, F. Löser, V.G. Lyssenko, K. Leo, S. Glutsch, F. Bechstedt, K. Köhler, Physica B 272, 180 (1999).

[5] J. Kastrup, R. Klann, H.T. Grahn, K. Ploog, L.L. Bonilla, J. Galan, M. Kindelan, M. Moscoso, R. Merlin, Phys. Rev. B 55, 2476 (1997).

[6] J. Kastrup, R. Klann, H.T. Grahn, K. Ploog, L.L. Bonilla, J. Galan, M. Kindelan, M. Moscoso, R. Merlin, Phys. Rev. B 52, 13761 (1995).

[7] S.A. Ktitorov, G.S. Simin, V.Y. Sindalovskii, Sov. Phys.-Solid State 13, 1872 (1972).

[8] A.A. Ignatov, K.F. Renk, E.P. Dodin, Phys. Rev. Lett. 70, 1996 (1993).

[9] H. Willenberg, G.H. Döhler, J. Faist, Phys. Rev. B 67, 085315 (2003).

[10] E. Schomburg, N.V. Demarina, K.F. Renk, Phys. Rev. B 67, 155302 (2003).

[11] A. Lisauskas, C. Blöser, R. Sachs, H.G. Roskos, A. Juozapavičius, G. Valušis, K. Köhler, submitted to Appl. Phys. Lett.

[12] L. Esaki, R. Tsu, IBM J. Res. Dev. 40, 61 (1970). 